\title{
MORPHOLOGICAL ANALYSIS OF ENGLISH COMPOUNDING IN JEEVA KLUI RESORT WEBSITE
}

\author{
Yessy Purnamasari \\ Universitas Suryakancana \\ yessy@unsur.ac.id
}

\begin{abstract}
In an English text, the variation of words are used in many kinds of wordformation. A text may consists of simple, compound, complex, or compoundcomplex sentences. There are also variation of phrases, clauses, or compounding to make the text becomes richer. However, when it comes to a text, those way cannot be applied in general. There are several aspects that distinguish whether it is a noun or it is a phrase. Thus, this research is expected to gives clear information in how a text in a website uses compounding as the element of its text. The research objectives are (1) to identify the kinds of compounding words found in the Jeeva Klui Resort's official website based on the part of of speech of its constituent and (2) to identify the kind of compounding words found in the Jeeva Klui Resort's official website based on the semantical relationship between the compound and its head. This research uses descriptive qualitative method which goal is a comprehensive summarization. The study focuses on the pronuciation of plural nouns in a text. The data, is taken from the official website of Jeeva Klui Resort which can be accessed through www.jeevaklui.com. For the linguistic studies, the theoretical benefit of this research is to give information about the use of English coumpounding in the text.
\end{abstract}

\section{INTRODUCTION}


The process of learning English can be done in many ways. As non-native speakers, there are several main skills in English to be comprehended: speaking, listening, writing, and reading. When it comes to understanding a context, for example a discourse analysis, some promblems might appear as there are certain rules that should be understood by the readers.

In an English text, the variation of words are used in many kinds of word-formation. A text may consists of simple, compound, complex, or compound-complex sentences. There are also variation of phrases, clauses, or compounding to make the text becomes richer.

Somehow, in understanding sentences, it is quite difficult to distinguish whether a sentence consist a phrase or a compound. The problems of determining whether it is a compound or a noun may occurs since they are somehow similar in some aspects. For example, greenhouse and green house are similar in writing and in a glance, we may think that both of them have no difference. However, the difficulty of differentiating between a phrase and a compound can be tackled by going back to the theories.

Delahunty and Garvey (2010) gives clear and simple definitions about the difference of compound and phrase. They said that in the compounds the main stress is on the first word; in the phrases the main stress is on the last word. While this pattern does not apply to all compounds, it is so generally true that it provides a very useful test.

However, when it comes to a text, those way cannot be applied in general. There are several aspects that distinguish whether it is a noun or it is a phrase. Thus, this research is expected to gives clear information in how a text in a website uses compounding as the element of its text by composing these objectives: a) to identify the kinds of compounding words found in the Jeeva Klui Resort's official website based on the part of of speech of its constituent and (b) t o identify the kind of compounding words found in the Jeeva Klui Resort's official website based on the semantical relationship between the compound and its head.

This research is basically a morphological analysis to find out what kind of compounding used in the introductory part of a website of a resort. Morphologically, the use of compound on the text can be seen from the part of speech of the compound and its constituent. Furthermore, in the semantic aspect, this research focuses on the semantical relationships between the compound and its head.

For the linguistic studies, the theoretical benefit of this research is to give information about the use of English coumpounding in the text. In addition, this research also contributes as the reference for another research. Moreover, this research is expected to be a useful 
source as the explanation written on this research will be from two sides:morphologically and semantically.

\section{THEORETICAL FRAMEWORK}

In a mophological analysis, it is important to understand the idea of what is word, base, root, affix, or stem. As Mowphology is defined as the study of the internal structure of words, deals with the forms of lexemes (inflection), and with the ways in which lexemes are formed 9word-formation) (Booij, 2005: 24), the beginning should be started from the idea of word.

\section{Morpheme}

Basically, words can be chopped into smaller pieces as Booij (2005) said that at the morphological level, words may consist of more than one unit as well which can be called as the morphological atoms of word.

Delahunty and Garvey (2010) defines morpheme as the smallest part of a word that has grammatical function or meaning. For example, sawed, sawn, sawing, and saws can all be analyzed into the morphemes $\{$ saw $\}+\{-$ ed $\},\{-n\},\{-$ ing $\}$, and $\{-s\}$, respectively. None of these last four can be further divided into meaningful units and each occurs in many other words, such as looked, mown, coughing, bakes.

Morpheme are divided into two types: free morpheme and bound morpheme. Morphemes can be divided into two general classes. Free morphemes are those which can stand alone as words of a language, whereas bound morphemes must be attached to other morphemes. Most roots in English are free morphemes (for example, dog, syntax, and to), although there are a few cases of roots (like -gruntle as in disgruntle) that must be combined with another bound morpheme in order to surface as an acceptable lexical item.

Free morphemes can be further subdivided into content words and function words. Content words, as their name suggests, carry most of the content of a sentence. Function words generally perform some kind of grammatical role, carrying little meaning of their own. One circumstance in which the distinction between function words and content words is useful is when one is inclined to keep wordiness to a minimum; for example, when drafting a telegram, where every word costs money. In such a circumstance, one tends to leave out most of the function words (like to, that, and, there, some, and but) concentrating instead on content words to convey the gist of the message. (Nordquist, 2018) 
To distinguish them, the following example may illustrates the difference between them simply:

$\begin{array}{llll}\text { Breaking } & \{\text { break }\}+\{\text {-ing }\} & \text { Hunters } & \text { \{hunter }\}+\{-\mathrm{s}\} \\ \text { Jumped } & \{\text { jump }\}+\{\text {-ed }\} & & \end{array}$

Those three word then be chopped into their smaller units and the sten can be identified into break, hunter, and jump. The stem of a word is the word form minus its inflectional affixes. $\{$-ing $\},\{-s\}$, and $\{$-ed $\}$ are the inflectional affixes to indicate the basic grammatical categories, for example tense of plurality. So, the free morphemes are break, hunter, and jump, in which they can stand by themself. Meanwhile, the bound morphemes from the above example are $\{$-ing $\},\{-\mathrm{s}\}$, and $\{$-ed $\}$. Their function are various either to give clarity whether the stem is a plural or in specific tenses. The main important thins is that bound morpheme cannto stand alone because they are not words. Thats why the bound morpheme must be attached to its free morpheme to make it becomes meaningful.

\section{Compounding}

There are many experts that gives definition about compounding. Plag (2002:170) states that: "compounding (sometimes also called composition) rather loosely as the combination of two words to form a new word. This definition contains two crucial assumptions, the first being that compounds consist of two (and not more) elements, the second being that these elements are words."

Based on the definition, Plag further give example about compounding as follows: astrophysics, biochemistry, photoionize, parks commissioner, teeth marks, systems analyst, pipe-and-slipper husband, off-the-rack dress, over-the-fence gossip.

Another definition is given by Delahunty and Garvey (2010). They defines that compound is: "In ordinary English spelling, compounds are sometimes spelled as single words, as in sawmill, sawdust; sometimes the parts are connected by a hyphen, as in jig-saw; and sometimes they are spelled as two words, as in chain saw, oil well. (Dictionaries may differ in their spellings.)"

Based on those two definitions, it can be concluded that the idea of compounding is actually when two or more words combined and form a new word with new meaning.

\section{The difference between phrase and compounding}


A phrase and compound are somehow similar in a glance. However, Delahunty and Garvey (2010) said that there are four ways to differ whether the words are a phrase or compound.First, the stress pattern of the compound word is usually different from the stress pattern in the phrase composed of the same words in the same order. In a compound, the stress is on the first word; however, in a phrase, the stress is on the last word. Second, the meaning of the compound may differ to a greater or lesser degree from that of the corresponding phrase. For example, the word blackbird is a species of bird whatever the color is, meanwhile in a phrase, it is a bird which color is black. So, because the meanings of compounds are not always predictable from the meanings of their constituents, dictionaries often provide individual entries for them. They do not do this for phrases, unless the meaning of the phrase is idiomatic and therefore not derivable from the meanings of its parts and how they are put together. Third, in many compounds, the order of the constituent words is different from that in the corresponding phrase. Fourth, in compounding, there is no modification allowed to the first element. Meanwhile, in noun phrases, it is allowed to do modification to the modifier: For example, since a blackbird is a compound, it is impossible to be modified by putting 'really' since gramatically it is not acceptable. Meanwhile, in a phrase, it is acceptable to put ' really' to a really black bird and gramatically, the meaning is acceptable.

\section{Art of Speech of Compound and Part of Speech of Its}

\section{Constituent}

Bauer (1983) as cited in Delahunty and Garvey (2010) said that there are a number of ways of approaching the study and classification of compound words, the most accessible of which is to classify them according to the part of speech of the compound and then sub-classify them according to the parts of speech of its constituents. There are compound nouns, compound verbs, compound adjectives, compound adverbs, and neo-classical compound.

\section{Semantical Relationship between a Compound and Its Head}

Linguists distinguish at least three different semantic relations between the head and modifier(s) of compounds as explained by Delahunty and Garvey (2010). First, endocentric compound is the type of compound which represents a subtype of whatever the head represents. That is, the head names the type, and the compound names the subtype. For example: a teapot is a kind of pot. 
Second, exocentric compound is the type of compound names a subtype, but the type is not represented by either the head or the modifier in the compound. For example, pickpocket represent type of people by denoting some distinguishing characteristic. There is typically another word, not included in the compound, that represents the type of which the compound represents the subtype.

Third, coordinative compound is the type in which both elements are heads; each contributes equally to the meaning of the whole and neither is subordinate to the other, for instance, bitter-sweet. Compounds like these can be paraphrased as both $\mathrm{X}$ and $\mathrm{Y}$, e.g., "bitter and sweet." Other examples include teacher-researcher and producer-director.

\section{METHOD}

This research uses descriptive qualitative method which goal is a comprehensive summarization (Lambert \& Lambert, 2012). The study focuses on the pronuciation of plural nouns in a text. The text, which becomes the data source, is taken from the official website of Jeeva Klui Resort which can be accessed through www.jeevaklui.com. The whole text is marked, copied, analysed into a specific list of nouns which have plural forms.

The analysis of English compounding in Jeeva Klui Resort official website is an effort to see the application of compound words in a text. The text, which is being analyzed and becomes the main data, focuses on the introductory part of the website. Since this research is a descriptive one, there will be description and explanations of the findings. In analyzing the data, there are several steps done by the writer: listing the sentences found in the introductory part of Jeeva Klui Resort website and organized them into a table, finding out the compound words in the introductory part of Jeeva Klui Resort website and organized them into a table, classifiying each compound words found in the introductory part of Jeeva Klui Resort website and organized them into a table and describing each compound word with the explanation of each category and its semantical relationship.

This research uses the introductory part of Jeeva Klui Resort's official website. It is a resort located in Lombok, West Nusa Tenggara and Bali which achieved the best tourist resort and hotel in Lombok by TripAdvisor, a website which specialized in sharing and giving information about many kinds of hotels and resorts around the world based on the personal experience of the guests. This resort develops the website in English since the guests are not only from Indonesia, but most of them are foreign tourists who want to spend their vacation time in Lombok. In this research, the text becomes the main data source and compound words found in the text will then be analyzed in order to find the result. 


\section{FINDINGS AND DISCUSSION}

This research is aimed to find out the kinds of compounding words found in the Jeeva Klui Resort's official website based on the part of of speech of its constituent and to identify the kind of compounding words found in the Jeeva Klui Resort's official website based on the semantical relationship between the compound and its head. In this chapter, the data available which is a full text then be identified by the compounding. The next step is then categorizing the following compound words into their types by the part of speech of its constituent and their semantic relations:

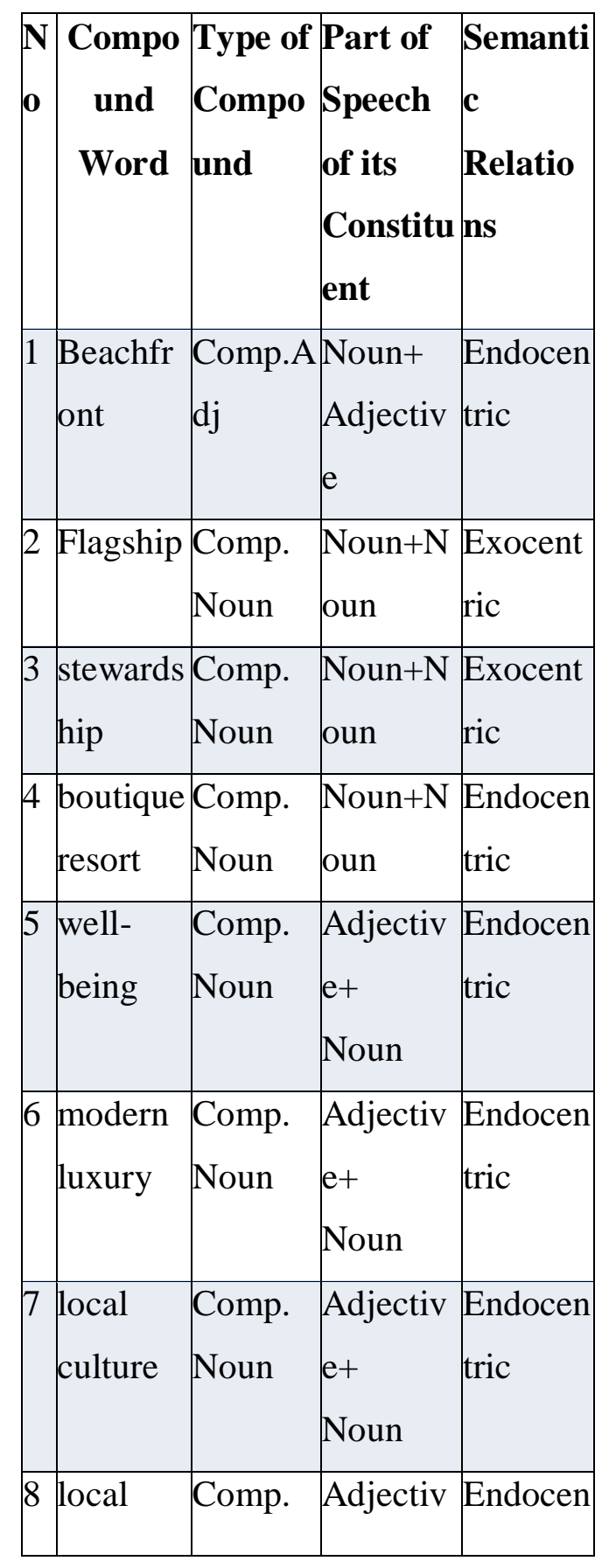




\begin{tabular}{|l|l|l|l|l|}
\hline & commun & Noun & e+ & tric \\
ities & & Noun & \\
\hline 9 & architect & Comp. & Adjectiv & Endocen \\
ural & Noun & e+ & tric \\
tradition & & Noun & \\
\hline 1 & cultural & Comp. & Adjectiv & Endocen \\
0 & diversity & Noun & e+ & tric \\
\hline 1 & exempla & Comp. & Adjectiv & Endocen \\
1 & ry & Noun & e+ & tric \\
\hline
\end{tabular}

The further analysis of each compound is explained as follows:

\section{a. Beachfront}

It is a compound adjective as the combination of noun+adjective. It is categorized as the endocentric compound since it has a head which expresses the core meaning of the compound, and it belongs to the same lexical category as the compound as the whole.

$$
\begin{gathered}
\text { beachfront [Adj] } \\
= \\
\text { beach }[\mathrm{N}]+\text { front }[\text { Adj] }
\end{gathered}
$$

b. Flagship

It is a compound noun as the combination of noun+noun. It is categorized as the exocentric compound since the semantic head is 'outside' (exo) the compound.

$$
\begin{gathered}
\text { flagship }[\mathrm{N}] \\
\qquad= \\
\text { flag }[\mathrm{N}]+\operatorname{ship}[\mathrm{N}]
\end{gathered}
$$

\section{c. Stewardship}

It is a compound noun as the combination of noun+noun. It is categorized as the exocentric compound since the semantic head is 'outside' (exo) the compound. 
steward $[\mathrm{N}]+\operatorname{ship}[\mathrm{N}]$

\section{d. Boutique resort}

It is a compound noun as the combination of noun+noun. It is categorized as the endocentric compound since it has a head which expresses the core meaning of the compound, and it belongs to the same lexical category as the compound as the whole.

$$
\begin{gathered}
\text { boutique resort }[\mathrm{N}] \\
\qquad= \\
\text { boutique }[\mathrm{N}]+\operatorname{resort}[\mathrm{N}]
\end{gathered}
$$

\section{e. Well-being}

It is a compound noun as the combination of adjective+noun. It is categorized as the endocentric compound since it has a head which expresses the core meaning of the compound, and it belongs to the same lexical category as the compound as the whole.

$$
\begin{gathered}
\text { well-being }[\mathrm{N}] \\
= \\
\text { well }[\text { Adj }]+\text { being }[\mathrm{N}]
\end{gathered}
$$

\section{f. Modern luxury}

It is a compound noun as the combination of adjective+noun. It is categorized as the endocentric compound since it has a head which expresses the core meaning of the compound, and it belongs to the same lexical category as the compound as the whole.

$$
\begin{gathered}
\text { modern luxury }[\mathrm{N}] \\
= \\
\text { modern }[\text { Adj }]+\text { luxury }[\mathrm{N}]
\end{gathered}
$$

\section{g. Local culture}

It is a compound noun as the combination of adjective+noun. It is categorized as the endocentric compound since it has a head which expresses the core meaning of the compound, and it belongs to the same lexical category as the compound as the whole.

\section{local culture $[\mathrm{N}]$}




$$
\text { local }[\text { Adj] + culture }[\mathrm{N}]
$$

\section{h. Local communities}

It is a compound noun as the combination of adjective+noun. It is categorized as the endocentric compound since it has a head which expresses the core meaning of the compound, and it belongs to the same lexical category as the compound as the whole.

$$
\begin{gathered}
\text { local communities }[\mathrm{N}] \\
\qquad= \\
\text { local }[\text { Adj }]+\text { communities }[\mathrm{N}]
\end{gathered}
$$

\section{i. Architectural traditions}

It is a compound noun as the combination of adjective+noun. It is categorized as the endocentric compound since it has a head which expresses the core meaning of the compound, and it belongs to the same lexical category as the compound as the whole.

$$
\begin{gathered}
\text { architectural tradition }[\mathrm{N}] \\
\qquad= \\
\text { architectural }[\mathrm{Adj}]+\operatorname{tradition}[\mathrm{N}]
\end{gathered}
$$

\section{j. Cultural diversity}

It is a compound noun as the combination of adjective+noun. It is categorized as the endocentric compound since it has a head which expresses the core meaning of the compound, and it belongs to the same lexical category as the compound as the whole.

$$
\begin{gathered}
\text { cultural diversity }[\mathrm{N}] \\
= \\
\text { cultural }[\text { Adj] +diversity }[\mathrm{N}]
\end{gathered}
$$

\section{k. Exemplary service}

It is a compound noun as the combination of adjective+noun. It is categorized as the endocentric compound since it has a head which expresses the core meaning of the compound, and it belongs to the same lexical category as the compound as the whole.

$$
\begin{gathered}
\text { exemplary service }[\mathrm{N}] \\
= \\
\text { exemplary }[\mathrm{Ad} \mathrm{j}]+\operatorname{service}[\mathrm{N}]
\end{gathered}
$$




\section{CONCLUSIONS}

The study of Morphology can be applied in many kinds of media, since Morphology defines the idea of the formation of word. In this research, the media that becomes the data is the introductort part of Jeeva Klui Resort Website. This part is using English as the language and there are many things can be analyzed from the morpohlogical aspect. Thus, the writer decides to analyze the use of English compounding in the text of the introductory part.

According to the discussions that has been done in the previous chapter, it can be concluded that there are 9 (nine) sentences found in the text which varies from simple into compound-complex. Based on the part of of speech of its constituent, there are 11 (eleven) compound words used in the text and amost all of them (91\%) is categorized as compound noun while there is only 1 (one) compound adjective. Based on the semantical relationship, there are 9 (nine) endocentric compounds and only 2 (two) which are categorized as exocentric compound.

\section{REFERENCES}

Aryati, Maharani Sri. (2014). An analysis of derivational affixes in the land of five towers novel by a.fuadi translated by angie kilbane. Kudus: Muria Kudus University

Booij, Geert. (2005). The grammar of words. United Stated: Oxford University Press

Delahunty, Gerald P., and James J Garvey. (2010). The English Language: From Sound to Sense. Perspectives on Writing. Fort Collins, Colorado: The WAC Clearinghouse and Parlor Press. Available http://wac.colostate.edu/books/sound/

Nordquist, Richard. Free Morphemes in English Definition and Examples.

ThoughtCo, Jun. 27, 2018, thoughtco.com/free-morpheme-words-and-word-parts1690872.11

Plag, Ingo. (2002). Word-formation in English. Cambridge: Cambridge University Press 